\title{
Medical Image of the Week: REM without Atonia
}

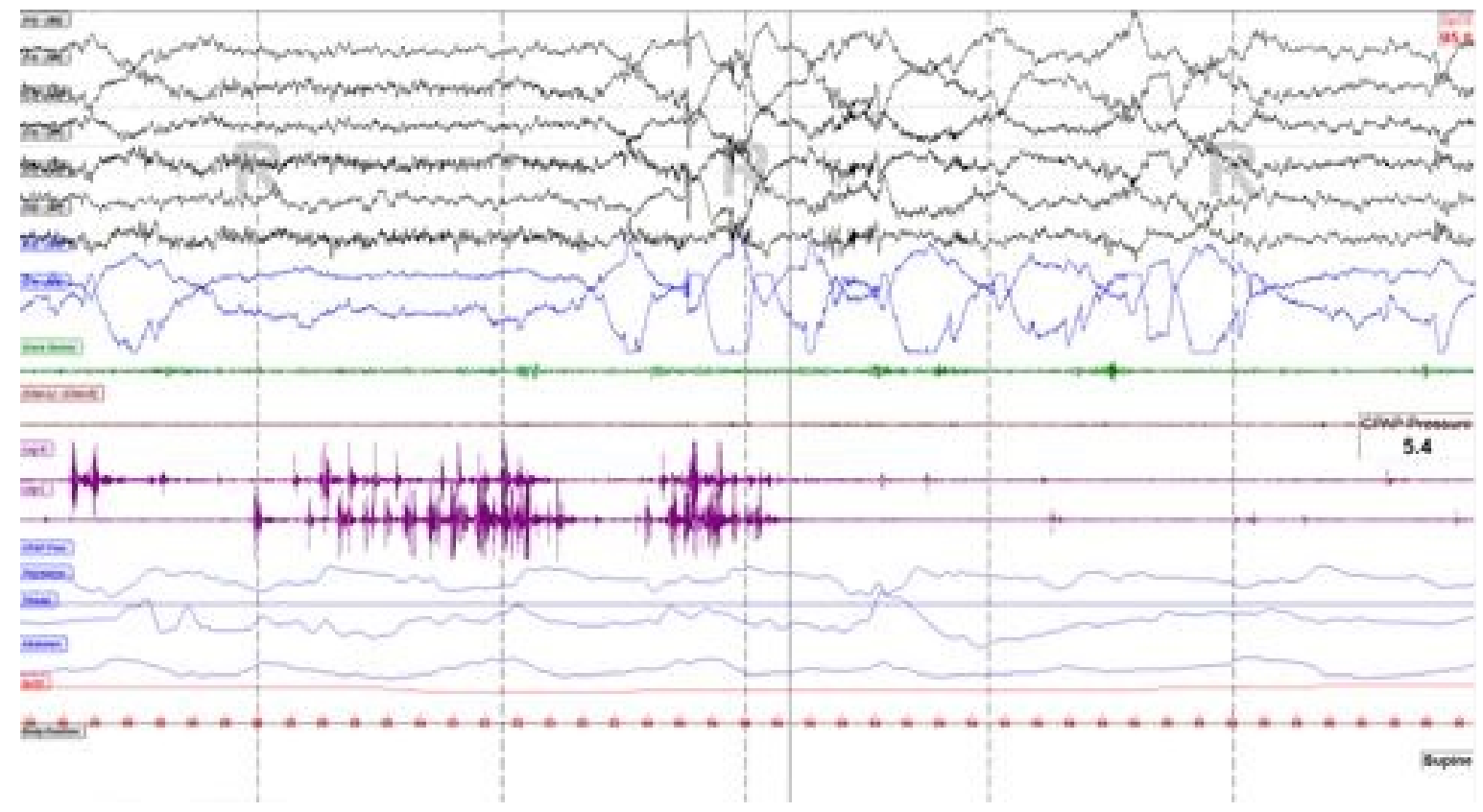

Figure 1. Polysomnogram with thirty-second epoch showing leg movements and relative increase in chin tone during REM sleep.

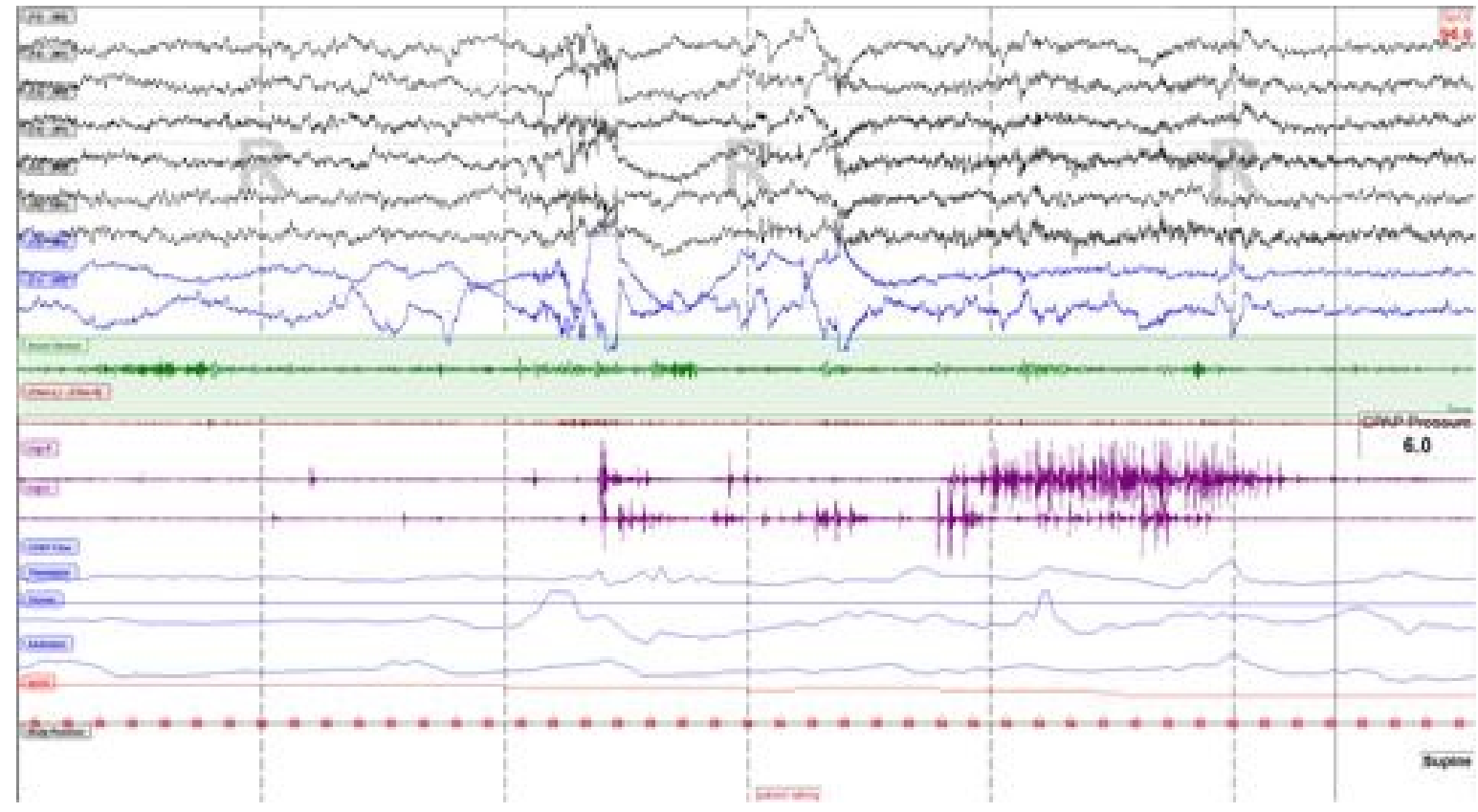

Figure 2. Polysomnogram with thirty-second epoch demonstrating similar interval of REM without atonia with the addition of sleep-talking. 
A 78 year-old man with a past medical history of Parkinson's disease (PD) presented to the sleep medicine clinic for evaluation of obstructive sleep apnea (OSA). An overnight polysomnogram (PSG) study was consistent with sleep apnea and revealed frequent leg and arm movements and sleep-talking during rapid eye movement (REM) sleep.

REM sleep behavior disorder (RBD) is a parasomnia characterized by repeated episodes of abnormal behavior occurring during REM sleep $(1,2)$. On PSG, REM sleep without atonia is seen while features of "normal REM" such as number of REM periods and REM cycling remain intact (2). RBD emerges most often in the context of alphasynucleinopathies, and occurs in up to $60 \%$ of PD patients (3). The presence of RBD may be an important preclinical symptom prior to the onset of PD. Cases of PD with RBD are associated with a unique phenotype with an older age of onset, longer disease duration, more profound motor disability, and greater degrees of hallucinations and cognitive dysfunction (3). Establishing a safe sleep environment can be of primary importance in patients with RBD as REM without atonia is associated with injurious behavior. Melatonin is effective as a first-line agent in patients with dementia. In nondemented patients without OSA, low-dose clonazepam is the first line intervention and is rarely associated with withdrawal or need for dose escalation (1). Treating concomitant OSA is important adjunctive therapy.

Jared Bartell, Safal Shetty MD, and Kenneth S. Knox MD University of Arizona Medical Center

Tucson, AZ

\section{References}

1. Aurora RN, Zak RS, Maganti RK, Auerbach SH, Casey KR, Chowdhuri S, Karippot A, Ramar K, Kristo DA, Morgenthaler TI; Standards of Practice Committee; American Academy of Sleep Medicine. Best practice guide for the treatment of REM sleep behavior disorder (RBD). J Clin Sleep Med. 2010;6(1):85-95. [PubMed]

2. Schenck CH, Mahowald MW. REM sleep behavior disorder: clinical, developmental, and neuroscience perspectives 16 years after its formal identification in SLEEP. Sleep. 2002;25(2):120-38. [PubMed]

3. Kim YE, Jeon BS. Clinical implication of REM sleep behavior disorder in Parkinson's disease. Parkinsons Dis. 2014;4(2):237-44. [CrossRef] [PubMed] 